\title{
Are Food Constituents Relevant to the Irritable Bowel Syndrome in Young Adults? \\ - A Rome III Based Prevalence Study of the Korean Medical Students
}

\author{
Hyun Joo Jung, Moo In Park, ${ }^{*}$ Won Moon, Seun Ja Park, Hyung Hun Kim, Eun Ji Noh, Gyu Jin Lee, Joo Hoon Kim and \\ Dong Gyu Kim \\ Department of Internal Medicine, Kosin University, College of Medicine, Busan, Korea
}

\begin{abstract}
Background/Aims
Irritable bowel syndrome (IBS) is prevalent in general population. This study investigates the prevalence of IBS in medical college students in Korea as well as the influence of dietary habits and nutritional intake on IBS.
\end{abstract}

\section{Methods}

This study is a cross-sectional study of 319 students (239 males and 80 females, age $22.3 \pm 2.5$ years) from the 6 grade levels of the Medical College in Korea. All students filled out a self-reported questionnaire for ROME III criteria. They also completed a questionnaire to validate dietary habits and food frequency in Korean.

\section{Results}

The overall prevalence of IBS was 29.2\% without correlation to age, body mass index and grade level in Medical School. However, the prevalence was significantly higher in females than males (33/80 vs 60/239, $P=0.007$ ). There were no significant differences between the IBS-group and the non-IBS group in aspect of nutrition. Not only the diet habits, but also the daily nutritional intake, and even the breakdown into the 12 micronutrients, yielded no significant differences between the 2 groups.

\section{Conclusions}

Twenty-nine percent of the medical college students have IBS with a greater prevalence in females. The dietary habits and nutritional intake of the students might not be associated with IBS.

\section{(J Neurogastroenterol Motil 2011;17:294-299)}

Key Words

Food habits; Irritable bowel syndrome; Prevalence

Received: January 1, 2011 Revised: May 20, 2011 Accepted: May 23, 2011

(c) This is an Open Access article distributed under the terms of the Creative Commons Attribution Non-Commercial License (http://creativecommons. org/licenses/by-nc/3.0) which permits unrestricted non-commercial use, distribution, and reproduction in any medium, provided the original work is properly cited.

*Correspondence: Moo In Park, MD

Department of Internal Medicine, College of Medicine, Kosin University, 34 Amnam-dong, Seo-gu, Busan 602-702, Korea

Tel: +82-51-990-6719, Fax: +82-51-990-3005, E-mail: mipark@ns.kosinmed.or.kr

Financial support: This study was supported in part by Kosin Institute for Medicine.

Conflicts of interest: None. 


\section{Introduction}

Irritable bowel syndrome (IBS), one of the commonly presented functional gastro-intestinal disorders, ${ }^{1}$ has a reported prevalence of $3 \%-22 \%$ in most Western countries ${ }^{2}$ and $5 \%-10 \%$ in most of Asia. ${ }^{3}$ This prevalence of IBS is affected by the diagnostic criteria used.

Although IBS is characterized with abdominal pain and altered bowel activity, it lacks any pathological organic changes. These symptoms are most frequently employed in making a differential diagnosis with the Rome III criteria. ${ }^{4}$

Generally, race, ${ }^{5}$ gender, ${ }^{6-9}$ age, ${ }^{10-12}$ marital status, ${ }^{13,14}$ stress, ${ }^{6-8,13-16}$ food, ${ }^{11,14,17-19}$ or alcohol and tobacco use ${ }^{11}$ have been considered as risk factors to IBS. In particular, the food or nutritional intake has been estimated to be closely associated with the pathophysiology of IBS. ${ }^{19}$ Several IBS studies have been performed since the publication of the Rome III criteria in 2006. ${ }^{1,4,20-22}$ However, there are only limited number of studies regarding the prevalence of IBS assessed by the Rome III criteria in not only the Korean general population, but also in a group of medical students. There are fewer reports about the relationship between dietary intake and gastrointestinal symptoms in well designed systematic research. $^{18,23}$

We investigated the prevalence of IBS in medical college students in Korea using the Rome III criteria for diagnosis. The influence of dietary habits and nutritional intake on IBS was also reported.

\section{Materials and Methods}

\section{Subjects}

A total of 319 students (2 grade levels of premedical courses and 3 grade levels of medical courses) of Kosin University, Medical College in Busan, Korea were studied in the winter of 2008. Actually, the medical course consists of 4 grade levels, but the fourth graders were excluded in this study because of their academic calendar. All students were asked to complete a self-reported questionnaire using the ROME III criteria. The questionnaire was a self translation of English version. They also validated their dietary habits and food frequency via a questionnaire in Korean. We obtained the informed consent from all the participants. Every analysis and questionnaire was carried out in accordance with appropriate Institutional Review Board regulations and approval.

\section{Study Protocol}

The first questionnaire (Rome III questionnaire) had 29 items using the Rome III criteria. ${ }^{24}$ There were several general items such as gender, age, height and weight. More specific items about the presence and frequency of abdominal pain or discomfort, its onset, the correlation between frequency or form of the stool and the pain, and improvement of the symptoms after defecation were also included. The diagnosis of IBS required that the student reported not only abdominal pain or discomfort at least 3 days a month in the last 3 months, but also at least 2 of the following 3 symptoms: relief of pain or discomfort after defecation, onset of pain or discomfort associated with a change of frequency of stool, and a change of appearance of stool.

The second questionnaire (Food Frequency Questionnaire) had 14 items to validate dietary habits along with alcohol and tobacco use. ${ }^{25}$ These items included not only the frequency, regularity and speed of consumption of the meal, but also the frequency of the oily, caffeinated, salted or fast food intake. Other items included the student's living situation such as living with family or apart from family as well as income or pocket money for a month. Another question inquired if the student had the average intake during 1 year in about 109 foods, which were common foods in Korea. Then, the nutritional value of each item was determined. These values included not only the amount of energy, protein, fat and carbohydrate, but also the amounts of calcium, phosphate, iron, zinc, vitamin A, B1, B2, B6, C, E, niacin and folate. The dietary consumption was evaluated as being either low, adequate or high as compared to the Korean recommended dietary allowance.

\section{Statistical Methods}

We compared the grade level, gender, body mass index and dietary habits as well as each nutritional factor between the non-IBS and IBS group using the Chi-squared test and Fisher's exact test. Data were expressed as frequency or mean with SD. $P$-values $<0.05$ were considered to indicate statistical significance. Statistical analyses were performed using a SPSS (Version 15.0 for Windows; SPSS Inc, Chicago, IL, USA).

\section{Results}

\section{Subjects Characteristics and Prevalence of Irritable Bowel Syndrome}

Of 480 students, 319 (66.5\%) completed the questionnaires. 
The mean age of included subjects was $22.3 \pm 2.5$ years (range 18-36) and 239 (74.9\%) were male.

Ninety-three students ( 60 male, 33 female) had symptoms of IBS. Therefore, overall prevalence of IBS was $29.2 \%$ without correlation to age, body mass index and grade level of medical course. The female group showed higher prevalence (33/80, $41.3 \%)$ than male $(60 / 239,25.1 \%)$. The prevalence of IBS among the 2 premedical level courses and 3 medical courses were $28 \%, 38 \%, 30 \%, 34 \%$ and $16 \%$, respectively, and showed no difference among each grade level (Table 1).

The IBS group was subdivided into diarrhea predominant (IBS-D), constipation predominant (IBS-C), mixed with diar-

Table 1. Irritable Bowel Syndrome Prevalence in Order of Characteristics of Students

\begin{tabular}{lcccc}
\hline & $\begin{array}{c}\text { Non-IBS } \\
(\mathrm{n}=226)\end{array}$ & $\begin{array}{c}\text { IBS group } \\
\left(\mathrm{n}=93[\%]^{\mathrm{a}}\right)\end{array}$ & $\begin{array}{c}\text { Total } \\
(\mathrm{N}=319)\end{array}$ & $P$-value \\
\hline $\begin{array}{l}\text { Grade of course } \\
\quad\end{array}$ & & & & $0.063^{\mathrm{b}}$ \\
$\begin{array}{l}\text { Premedical } \\
\text { Medical }\end{array}$ & 91 & $45(33.1)$ & 136 & \\
Gender & 135 & $48(16.4)$ & 183 & \\
$\quad$ Male & 179 & $60(25.1)$ & 239 & $0.007^{\mathrm{c}}$ \\
Female & 47 & $33(41.3)$ & 80 &
\end{tabular}

${ }^{\mathrm{a}}$ Prevalence of IBS, ${ }^{\mathrm{b}}$ Analysed by Pearson Chi-Square, ${ }^{\mathrm{c}}$ Analysed by Fisher's exact test.

IBS, irritable bowel syndrome. rhea and constipation (IBS-M), or undetermined categories (IBS-U) according to the bowel movement frequency and stool consistency. Among the 93 IBS students, the proportions of IBS-D, IBS-C, IBS-M and IBS-U were $23.6 \%$, 3.2\%, $70.9 \%$ and $2.2 \%$, respectively.

\section{Irritable Bowel Syndrome and Diet Habit or Nutritional Intake}

In the present study, the daily caloric intake with all the dietary factors showed no significant differences between IBS and non-IBS groups (absolute vs mean, 1,917 kcal vs 2,021 kcal). There were no significant differences between non-IBS group and IBS group, when the nutrient intake of each patient was divided into low, adequate or high by Korean's recommended dietary allowance (Table 2). No significant differences were detected in the \% ratio (14.6\%:21.9\%:63.4\% vs $14.7 \%: 22.7 \%: 62.6 \%$, respectively) of protein, lipid and carbohydrate in the daily nutrition.

\section{Discussion}

The prevalence of IBS shows great diversity. In Western countries, the IBS prevalence has been reported as $10 \%-24 \%$. ${ }^{2,26-31}$ However, in most of Asia, it has been reported as being 5\%-10\% lower than western countries, ${ }^{3}$ with an especially low prevalence of $7.85 \%$ among college and university students of North China. ${ }^{32}$

Table 2. Comparison of 2 Groups (Non-Irritable Bowel Syndrome and Irritable Bowel Syndrome) on Levels of Intake of Energy, Fat and 12 Micronutrients

\begin{tabular}{|c|c|c|c|c|c|c|c|}
\hline & \multicolumn{3}{|c|}{ Non-IBS $(\mathrm{n}=226)$} & \multicolumn{3}{|c|}{$\operatorname{IBS}(\mathrm{n}=93)$} & \multirow{2}{*}{$P$-value } \\
\hline & Lower (\%) & Adequate (\%) & Higher (\%) & Lower (\%) & Adequate $(\%)$ & Higher (\%) & \\
\hline Energy & $99(43.8)$ & $70(30.9)$ & $57(25.2)$ & $47(50.5)$ & $24(25.8)$ & $22(23.7)$ & 0.51 \\
\hline Fat & $55(24.3)$ & $103(45.5)$ & $68(30)$ & $23(24.7)$ & $43(46.2)$ & $27(29.0)$ & 0.98 \\
\hline Calcium & $139(61.5)$ & $64(28.3)$ & $23(10.1)$ & $60(64.5)$ & $25(26.9)$ & $8(8.6)$ & 0.85 \\
\hline Phosphate & $27(11.9)$ & $78(34.5)$ & $121(53.5)$ & $11(11.8)$ & $32(34.4)$ & $50(53.7)$ & 0.99 \\
\hline Iron & $113(50.0)$ & $76(33.6)$ & $37(16.3)$ & $53(57.0)$ & $30(32.3)$ & $10(10.7)$ & 0.35 \\
\hline Zinc & $129(57.1)$ & $74(32.7)$ & $23(10.1)$ & $54(58.1)$ & $30(32.3)$ & $9(9.6)$ & 0.98 \\
\hline Vt.A & $149(65.9)$ & $61(27.0)$ & $16(7.0)$ & $71(76.3)$ & $17(18.3)$ & $5(5.3)$ & 0.18 \\
\hline Vt. $B_{1}$ & $66(29.2)$ & $108(47.8)$ & $52(23.0)$ & $28(30.1)$ & $44(47.3)$ & $21(22.5)$ & 0.94 \\
\hline $\mathrm{Vt} \cdot \mathrm{B}_{2}$ & $112(49.6)$ & $84(37.2)$ & $30(13.2)$ & $50(53.8)$ & $30(32.6)$ & $13(13.9)$ & 0.70 \\
\hline $\mathrm{Vt} . \mathrm{B}_{6}$ & $53(23.5)$ & $97(42.9)$ & $76(33.6)$ & $24(25.8)$ & $42(45.2)$ & $27(29.0)$ & 0.72 \\
\hline Vt.C & $66(29.2)$ & $64(28.3)$ & $96(42.4)$ & $29(31.2)$ & $29(31.2)$ & $35(37.6)$ & 0.72 \\
\hline Vt.E & $80(35.4)$ & $90(39.8)$ & $56(24.7)$ & $36(38.7)$ & $35(37.6)$ & $22(23.6)$ & 0.85 \\
\hline Niacin & $66(29.2)$ & $99(43.8)$ & $61(26.9)$ & $27(29.0)$ & $40(43.0)$ & $26(27.9)$ & 0.98 \\
\hline Folate & $125(55.3)$ & $75(33.2)$ & $26(11.5)$ & $55(59.1)$ & $29(31.2)$ & $9(9.6)$ & 0.79 \\
\hline
\end{tabular}

IBS, irritable bowel syndrome. 
In Korea, the reported IBS prevalence in college and university students is $5.7 \%$, ${ }^{18}$ compared to $8.3 \%$ in those receiving a routine health check-up. ${ }^{33}$ The prevalence in the general Korean population has been reported to be between $6.6 \%-9.0 \% .^{11,21}$ The diversity of prevalence in IBS mainly depends on the used diagnostic criteria and the characteristics of the included subjects. Several reports have shown that the prevalence of IBS is significantly higher when using the Rome III criteria rather than the Rome II. $^{21,34,35}$

The reported prevalence of IBS by the Rome III criteria was 9.0\%-21.9\%. ${ }^{36}$ Our cross-sectional study showed $29.2 \%$ prevalence of IBS, determined on the basis of the Rome III criteria. This high prevalence was found in the medical college students. In a large scaled study in Europe, the highest prevalence (12.2\%) of IBS was in the 18-34 age group, ${ }^{30}$ while our student's mean age was $22.3 \pm 2.5$ years (range 18-36). And our subject population was not from the general population. In a study of healthy adults, which was performed in the medical students, they showed higher prevalence of IBS of $15.8 \%,{ }^{37}$ and suggested that the reason of the high prevalence would be from medical students, because these groups were highly stressed and more aware of somatic symptoms. The exact mechanism of psychological stress inducing abdominal symptoms has not been established yet. However, stress is an accepted contributing factor for IBS. $^{6-8,13-16}$ This effect of stress is presented as a conceptual model of the relationship between several factors and IBS. ${ }^{28}$ However, the level of stress in medical college students has not been expressed numerically. Therefore, it is uncertain that the reason of the high prevalence in our study was influenced by intensely stressful condition of medical school.

Many studies have reported that the prevalence of IBS is higher in females, and have suggested some hormonal effects for the etiology. ${ }^{17,38}$ Actually, in many reports, the IBS prevalence in female was higher than in male. ${ }^{29}$ In our study, female prevalence of IBS was higher. Though the incidence had statistical significance, the imbalance of gender ratio (male:female $=2.98: 1$ ) in our population is a limitation of our study.

Among several risk factors, dietary components are a small part of the pathophysiological complexion of IBS. ${ }^{28}$ However, it has been assumed to be associated with the pathophysiology of IBS including functional gastrointestinal disease in several studies. ${ }^{19,39}$ Fatty foods, beans, gas-producing foods, alcohol, caffeine and lactose (in lactose-deficient individuals) have been accepted as certain dietary substances that may aggravate gastrointestinal symptoms. ${ }^{28,40}$ It was reported that the high-fat diet has a significant correlation with IBS. ${ }^{41}$ Those people that prefer spicy and salty food had a 1.80 risk of developing IBS. ${ }^{23}$ And, there was a report of significance between the number of meals a day or types of families and IBS. ${ }^{18}$

In our study many parameters were evaluated. These items include the number of meals per day, the regularity of eating breakfast, daily meal times and the speed in consuming the food. Also included were the symptoms of lactose intolerance, the degree of saltiness of food, the frequency of overeating, fatty food intake, fast food intake and drinking caffeinated beverages. Alcohol and tobacco use, the amount of milk products ingested and hot soup or food intake were evaluated. The daily caloric intake, the energy amount ingested and the value of the micronutrients were also evaluated. However, we did not find any statistical significance between dietary pattern or nutritional intake and IBS. This result did not satisfy our expectation. It seems to be difficult to prove the causative relationship between IBS and dietary pattern or each nutritional factor. Moreover, since the questionnaire required the memory of dietary habits or kinds of food for a recent year, recall bias was unavoidable. And the small sample size may contribute to the insignificant results. If we had investigated much larger population, the results could have been different.

In spite of these limitations, this study is the first investigation regarding the prevalence of IBS in the Korean medical college students using the Rome III criteria. The use of the Food Frequency Questionnaire, in evaluating nutritional association with IBS is also the first.

More studies using the Rome III criteria are needed to evaluate the IBS prevalence in the Korean general population and specific groups. Since the distinct Korean foods or recipes differ greatly from Western cultures, a large scale cohort study regarding the dietary pattern or nutrients and etiology of IBS is essentially needed.

\section{References}

1. Drossman DA. The functional gastrointestinal disorders and the Rome III process. Gastroenterology 2006;130:1377-1390.

2. Oberndorff-Klein Woolthuis AH, Brummer RJ, de Wit NJ, Muris JW, Stockbruügger RW. Irritable bowel syndrome in general practice: an overview. Scand J Gastroenterol 2004;39(suppl):17-22.

3. Chang FY, Lu CL. Irritable bowel syndrome in the 21st century: perspectives from Asia or South-east Asia. J Gastroenterol Hepatol 2007;22:4-12.

4. Drossman DA, Douglas A, eds. Rome III: The functional gastrointestinal disorder. 3rd ed. McClean, Virginia: Degnon Associates 
2007:885-897.

5. Wigington WC, Johnson WD, Minocha A. Epidemiology of irritable bowel syndrome among African Americans as compared with whites: a population-based study. Clin Gastroenterol Hepatol 2005;3:647-653.

6. Park KS, Ahn SH, Hwang JS, et al. A survey about irritable bowel syndrome in South Korea: prevalence and observable organic abnormalities in IBS patients. Dig Dis Sci 2008;53:704-711.

7. Kumano H, Kaiya H, Yoshiuchi K, Yamanaka G, Sasaki T, Kuboki T. Comorbility of irritable bowel syndrome, panic disorder, and agoraphobia in a Japanese representative sample. Am J Gastroenterol 2004;99:370-376.

8. Boyce PM, Koloski NA, Talley NJ. Irritable bowel syndrome according to varying diagnostic criteria, are the new Rome II criteria unnecessarily restrictive for research and practice? Am J Gastroenterol 2000;95:3176-3183.

9. Gwee KA, Lu CL, Ghoshal UC. Epidemiology of irritable bowel syndrome in Asia: something old, something new, something borrowed. J Gastroenterol Hepatol 2009;24:1601-1607.

10. Gwee KA, Wee S, Wong ML, Png DJ. The prevalence, symptom characteristics, and impact of irritable bowel syndrome in an Asian urban community. Am J Gastroenterol 2004;99:924-931.

11. Han SH, Lee OY, Bae SC, et al. Prevalence of irritable bowel syndrome in Korea: population-based survey using the Rome II criteria. J Gastroenterol Hepatol 2006;21:1687-1692.

12. Miwa H. Prevalence of irritable bowel syndrome in Japan: internet survey using Rome III criteria. Patient Prefer Adherence 2008;2: 143-147.

13. Husain N, Chaudhry IB, Jafri F, Niaz SK, Tomenson B, Creed F. A population-based study of irritable bowel syndrome in a non-Western population. Neurogastroenterol Motil 2008;20:1022-1029.

14. Dong L, Dingguo L, Xiaoxing X, Hanming L. An epidemiologic study of irritable bowel syndrome in adolescents and children in China: a school-based study. Pediatrics 2005;116:e393-e396.

15. Xiong LS, Chen MH, Chen HX, Xu AG, Wang WA, Hu PJ. A population-based epidemiologic study of irritable bowel syndrome in South China: stratified randomized study by cluster sampling. Aliment Pharmacol Ther 2004;19:1217-1224.

16. Shen L, Kong H, Hou X. Prevalence of irritable bowel syndrome and its relationship with psychological stress status in Chinese university students. J Gastroenterol Hepatol 2009;24:1885-1890.

17. Pan G, Lu S, Ke M, Han S, Guo H, Fang X. Epidemiologic study of the irritable bowel syndrome in Beijing: stratified randomized study by cluster sampling. Chin Med J 2000;113:35-39.

18. Kim YJ, Ban DJ. Prevalence of irritable bowel syndrome, influence of lifestyle factors and bowel habits in Korean college students. Int J Nurs Stud 2005;42:247-254.

19. Moon W, Park MI. [Dietary Factors in Functional Gastrointestinal Disorders.] Kor J Neurogastroenterol Motil 2007;13:1-7. [Korean]

20. Thompson WG. The road to Rome. Gastroenterology 2006;130: 1552-1556.

21. Park DW, Lee OY, Shim SG, et al. The differences in prevalence and sociodemographic characteristics of irritable bowel syndrome according to Rome II and Rome III. J Neurogastroenterol Motil 2010;16:186-193.

22. Oliver G, Yoon SL. Irritable bowel syndrome: epidemiology, diag- nosis and treatment: An update for health-care practitioners. J Gastroenterol Hepatol 2010;25:691-699.

23. Kim JR, Urm SH, Chun JH, et al. Relationship between life style, the level of stress and irritable bowel syndrome on 1498 male white collars. Kor J Prev Med 1997;30:791-804. [Korean]

24. Longstreth GF, Thompson WG, Chey WD, Houghton LA, Mearin F, Spiller RC. Functional bowel disorders. Gastroenterol 2006;130:1480-1491.

25. Ahn Y, Kwon E, Shim JE, et al. Validation and reproducibility of food frequency questionnaire of Korean genome epidemiologic study. Eur J Clin Nutr 2007;61:1435-1441.

26. Agréus L, Svärdsudd K, Nyrén O, Tibblin G. Irritable bowel syndrome and dyspepsia in the general population: overlap and lack of stability over time. Gastroenterology 1995;109:671-680.

27. Müller-Lissner SA, Bollani S, Brummer RJ, et al. Epidemiological aspects of irritable bowel syndrome in Europe and North America. Digestion 2001;64:200-204.

28. Drossman DA, Camilleri M, Mayer EA, Whitehead WE. AGA technical review on irritable bowel syndrome. Gastroenterology 2002; 123:2108-2131.

29. Drossman DA, Li Z, Andruzzi E, et al. U.S. householder survey of functional gastrointestinal disorders. Prevalence, sociodemography, and health impact. Dig Dis Sci 1993;38:1569-1580.

30. Hungin AP, Whorwell PJ, Tack J, Mearin F. The prevalence, patterns and impact of irritable bowel syndrome: an international survey of 40,000 subjects. Aliment Pharmacol Ther 2003;17:643-650

31. Hungin AP, Chang L, Locke GR, Dennis EH, Barghout V. Irritable bowel syndrome in the United States: prevalence, symptom patterns and impact. Aliment Pharmacol Ther 2005;21:1365-1375.

32. Dong YY, Zuo XL, Li CQ, Yu YB, Zhao QJ, Li YQ. Prevalence of irritable bowel syndrome in Chinese college and university students assessed using Rome III criteria. World J Gastroenterol 2010;33: 4221-4226.

33. Hwang SY, Choi CH, Rhie SY, et al. [The Frequency and Related Factors of Irritable Bowel Syndrome in Health Check up Subjects.] Kor J Neurogastroenterol Motil 2006;12:136-143. [Korean]

34. Dai N, Cong Y, Yuan H. Prevalence of irritable bowel syndrome among undergraduates in Southeast China. Dig Liver Dis 2008;40: 418-424.

35. Sperber AD, Shvartzman P, Friger M, Fich A. A comparative reappraisal of the Rome II and Rome III diagnostic criteria: are we getting closer to the 'true' prevalence of irritable bowel syndrome? Eur J Gastroenterol Hepatol 2007; 19:441-447.

36. Chang FY, Lu CL, Chen TS. The current prevalence of irritable bowel syndrome in asia. J Neurogastroenterol Motil 2010;16:389400.

37. Tan YM, Goh KL, Muhidayah R, Ooi CL, Salem O. Prevalence of irritable bowel syndrome in young adult Malaysians: a survey among medical students. J Gastroenterol Hepatol 2003;18:1412-1416

38. Wald A, Van Thiel DH, Hoechstetter L, et al. Gastrointestinal transit: the effect of the menstrual cycle. Gastroenterology 1981;80: 1497-1500.

39. Feinle-Bisset C, Vozzo R, Horowitz M, et al. Diet, food intake, and disturbed physiology in the pathogenesis of symptoms in functional dyspepsia. Am J Gastroenterol 2004;99:170-181.

40. Zuo XL, Li YQ, Shi L, et al. Visceral hypersensitivity following cold 
water intake in subjects with irritable bowel syndrome. J Gastroenterol 2006;41:311-317

41. Saito YA. Locke GR 3rd, Weaver AL, Zinsmeister AR, Talley NJ.
Diet and functional gastrointestinal disorders: a population-based case-control study. Am J Gastroenterol 2005;100:2743-2748. 